\title{
Utilisation de paiement mobile au temps de crise et réduction des inégalités d'accès aux services
}

\author{
Bouchra BENYACOUB, Hanane AZIRAR \\ Faculté des sciences juridiques économiques et sociales, USMBA Fès, Maroc
}

\begin{abstract}
Résumé : L'inclusion financière a suscité au cours de ces dernières années un intérêt grandissant de la part des organismes internationaux, des régulateurs du secteur financier ainsi que des pouvoirs publics. Cette tendance traduit une prise de conscience du rôle que peuvent jouer les services financiers dans la promotion d'une croissance inclusive et la concrétisation des objectifs de développement durable notamment en favorisant la réduction de la pauvreté et des disparités entre les genres. Au Maroc plusieurs stratégies ont été mises en place pour réduire les inégalités d'accès aux services financiers. Avec le lancement du paiement mobile en 2016, les pouvoirs publics ambitionne de généraliser l'accès des populations défavorisées aux services financiers. L'objet de cette étude est d'évaluer l'effet du mobile money sur la réduction des inégalités d'accès aux services financiers numériques. L'éstimation d'un modèle composé de 7 variables sur un échantillon de 156 personnes au Maroc révèle que le mobile money réduit significativement les inégalités d'accès aux services financiers. En effet, il ressort que deux variables ont un effet significatif sur la réduction des inégalités d'accès aux services financiers à savoir l'âge et le revenu. Il ressort aussi que le manque de confiance, de provision et d'éducation financière jouent un rôle important dans l'exclusion financière.
\end{abstract}

Mots clés : mobile money, crise, réduction des inégalités, accès aux services financiers, inclusion financière.

\section{INTRODUCTION}

Selon les indicateurs de la banque mondiale, le taux de croissance des pays d'Afrique subsaharienne est passé de 5,5\% à 2,28\% entre l'année 2010 et 2019, ce ralentissement s'explique par la progression du taux de la pauvreté qui reste toujours très élevé dans cette région. $85 \%$ des pauvres de la planète vivent en Afrique subsaharienne et en Asie du sud, les $15 \%$ restants, soit environ 106 millions de personnes, vivaient dans les quatre autres régions du monde.

Dans ce contexte, la répartition non équitable de la richesse dans les pays de l'Afrique prouve qu'il y'a plus d'inégalités. Pour atténuer ce phénomène, plusieurs institutions et entités internationales (Banque Mondiale, FMI, PNUD, l'OCDE ...) présentent des stratégies de développement mais insistent principalement sur le développement financier qui est un facteur catalyseur dans la réduction de l'exclusion financière et de la réduction de l'inégalité. En effet, plusieurs travaux théoriques, appartenant principalement aux champs microéconomiques, ont montré les impacts économiques et sociaux de l'utilisation du mobile money

Au cours de la dernière décennie, le Maroc a élaboré plusieurs stratégies pour élargir l'accès aux différents services financiers au profit des différents segments de la population (particuliers et entreprises). En effet l'inclusion financière est devenue une nécessité pour le développement, en particulier l'utilisation de services 
financiers numériques à travers l'utilisation de téléphone mobile ou d'un smartphone, l'introduction par Bank Al Maghrib des établissements de paiement au niveau de la loi bancaire, permettra la construction d'un système financier inclusif et durable. Cependant, la crise sanitaire covid-19, avec ses lourdes conséquences et implications économiques et sociales, a entrainé l'exclusion financière d'un nombre important de citoyens. L'utilisation du mobile money est devenue une nécessité pour permettre la distribution des aides publiques aux populations touchées par la crise sanitaire, mais aussi pour le maintien de la vie économique et le renforcement du lien social.

L'objectif cet article de répondre aux questions suivantes: Est-ce que la digitalisation financière des services financiers réduit l'exclusion financière des populations défavorisées ? Est-ce que la crise du covid-19 a accéléré l'utilisation du mobile ?

Pour répondre à ce questionnement, nous allons faire une comparaison de l'utilisation de mobile money avant la crise et après la crise à travers une étude qui porte sur un échantillon de 170 personnes, à fin de déterminer l'effet de la crise sur le paiement mobile et son effet sur la réduction des inégalités au Maroc.

\section{LE PAIEMENT MOBILE AU MAROC}

Le mobile Banking, au sens strict du terme, désigne les services financiers par téléphone portable, offerts par les banques (Brack 2013). Il s'agit principalement dans ce cas de services de consultation de solde, de paiement de factures et de transfert d'argent. Au sens large, le concept s'étend à l'ensemble des services financiers pouvant être offerts avec ou sans compte bancaire par tout établissement agréé à cet effet par SMS ou via des applications sur Smartphone. C'est « un transfert de fonds, en échange d'un bien ou service, où le téléphone portable est utilisé à l'initiation et à la confirmation du paiement » (Tobbin, 2011). Donc le mobile money est un nouveau mode de paiement, c'est un service financier par l'utilisation du téléphone portable, offert par les institutions financières, il passe à travers les réseaux des opérateurs mobiles et il recouvre plusieurs types de paiements à travers les canaux d'internet. Il consiste à effectuer différentes opérations financières à partir du téléphone mobile et elles prennent deux formes essentielles qui sont principalement l'échange d'informations financières et l'exécution des transactions financières.

Le mobile money est parmi les outils technologiques qui favorisent une grande pénétration d'épargne. Dans ce sens, plusieurs travaux d'Asongu (2018) ont montré que l'utilisation de mobile money réduit les inégalités de revenus dans les pays en développement. C'est un moyen qui favorise l'inclusion financière et permet aux agents économiques (particuliers ou entreprises) d'accéder facilement aux services financiers à partir des applications spécifiques à cette finalité accessible à tout moment quelle que soit leurs zones de résidence.

Le Mobile Paiement a été dans plusieurs pays un vecteur important, voire central, d'inclusion financière. Il a permis d'atteindre des niveaux très élevés de pénétration du compte et a été positionné pour une part significative des populations comme le principal vecteur de «financiarisation ». Le succès de ce modèle s'explique par l'attractivité de la proposition de valeur pour les usagers, en particulier pour les ménages les moins aisés :

- Une forte capillarité grâce au développement de réseaux d'agents permettant aux usagers de réaliser leurs opérations dans un nombre important de points de contact ;

- La simplicité des services et de leur utilisation permettant un usage facile même pour les populations ayant des connaissances limitées des produits financiers ; 
- La compétitivité du prix, significativement plus faible par rapport aux prix des opérations classiques ;

- Les conditions moins restrictives d'accès pour les comptes avec une procédure « Know Your Customer » plus légère par rapport aux comptes classiques.

Depuis quelques années Bank Al Maghrib a mis l'inclusion financière des segments vulnérables de la population (femmes, TPME, les jeunes) parmi les leviers de développement économique et sociale du pays. consciente des opportunités offertes par la digitalisation pour la promotion de l'inclusion financière et de la forte pénétration de la téléphonie mobile aussi bien en milieu urbain que rural, BAM s'est engagée depuis plusieurs années dans une stratégie de développement du Mobile Paiement. En effet, une première étape avait été franchie en 2014 après avoir introduit les établissements de paiement en tant que nouvelle catégorie d'acteurs du secteur financier au niveau de la loi bancaire 102-12. Ces institutions sont capables, en complément des établissements de Crédit, d'ouvrir des comptes de paiement, de collecter des dépôts et d'offrir des services de paiement à leur clientèle favorisant ainsi l'innovation et la création d'un marché concurrentiel.

Depuis, le secteur financier s'est mobilisé sous l'impulsion de BAM et a poursuivi les travaux de mise en place d'une solution nationale de Paiement Mobile Interopérable largement diffusée et à bas coût. En effet, un comité stratégique se composant, outre BAM et l'ANRT, les principales banques de la place, les trois opérateurs télécoms, le MEFRA et le Ministère de l'Industrie, a été mis en place pour piloter ce projet. Ce comité a été chargé de définir les règles nécessaires au bon fonctionnement des transactions inter-opérées, de mener la syndication et d'affiner le modèle économique pour un lancement effectif de la solution. Parallèlement, BAM a octroyé les agréments aux établissements de paiement dont les dossiers ont été examinés et évalués conformément aux exigences légales et réglementaires. Ces établissements reposent sur des agents principaux et détaillants leur permettant de renforcer la capillarité de leurs réseaux et de servir les segments sous-desservis voire exclus du secteur formel.

Par ailleurs l'objectif de la finance inclusive va au-delà d'une simple combinaison entre le taux de bancarisation et le taux de pénétration des lignes télécoms. Le modèle inclusif a un enjeu stratégique : Accélérer la généralisation de l'usage des services financiers digitaux. Par exemple, l'opérateur Inwi a été le premier à déployer le mobile money au Maroc en septembre 2019.

Parmi les avantages de mobile money on trouve: Un faible coût, l'instantanéité et une large diffusion. Autrement dit, la digitalisation garantit systématiquement la levée de nombreux freins à travers des services transactionnels à faibles coûts. Le mobile money permet donc de faciliter l'accès aux services financiers, il consiste en des transactions bancaires effectuées depuis un téléphone mobile et permet ainsi de lever les barrières imposées par les circuits classiques qui pénalisent une large clientèle défavorisée en particulier les femmes et les jeunes ainsi que les TPME.

\section{LA CRISE SANITAIRE ET L'UTILISATION DE MOBILE MONEY}

L'apparition de la pandémie du Covid-19 et sa propagation dans le monde a eu des répercussions déplorables sur la santé des citoyens et leur bien-être, sur l'activité économique, sur les revenus des ménages, ainsi que sur les budgets des Etats. La crainte d'une contagion incontrôlée de la population a poussé les responsables dans plusieurs pays à prendre des mesures préventives consistant à fermer leurs frontières, à restreindre d'une manière sévère les mouvements des personnes en leur imposant des règles de conduite stricte. Les conséquences déplorables sur le plan économique et social de cette crise sanitaire ont entrainé l'exclusion financière d'un nombre important de citoyens. 
Cette pandémie a poussé beaucoup des pays à renforcer la technologie pour résoudre des contraintes associées aux mesures restrictives imposées la pandémie. Le Wali de Bank Al Maghrib avait déclaré que la liquidité bancaire a été impactée par les sorties de cash consécutives aux aides accordées par l'État aux ménages durant cette crise sanitaire et que s'il y avait le mobile banking, on aurait pu limiter cette situation

Selon une étude mené par le fond monétaire international (FMI),l'argent mobile est un moyen efficace, et conforme aux règles de distanciation physique, pour effectuer des transferts monétaires à grande échelle, compte tenu du nombre très élevé de détenteurs et d'utilisateurs de téléphones mobiles dans les pays émergents et les pays en développement et du fait que, mondialement, il existe 228 points de services d'argent mobile (de petits commerces où les clients peuvent déposer ou retirer des fonds de leur compte mobile, ou acheter à crédit) pour 100000 adultes, contre seulement 11 banques et 33 distributeurs automatiques de billets. Donc l'argent mobile peut aider les agents qui habitent aux zones rurales d'effectuer leurs transactions sans déplacement. Donc la technologie est devenue la solution pour améliorer l'accès aux services financiers et réduire les coûts par l'utilisation de de mobile money.

En effet, la crise sanitaire du covid-19 a été accompagnée d'une accélération de la technologie au niveau de tous les secteurs principalement les secteurs publics. Il est donc utile de souligner ici l'apport considérable du mobile money durant la période du confinement, un moyen indispensable, notamment pour maintenir la vie économique et le lien social, voire un accélérateur de solidarité via la distribution des aides publiques aux populations vulnérables, celles touchées durement par la crise. Selon BAM près de 1.5 million de mobile Wallets opérationnels à fin septembre 2020, ce chiffre reste insuffisant pour assurer l'inclusion financière des populations défavorisées. Le Maroc est donc appelé à accélérer ce chantier pour inclure les couches de la population qui sont exclues du système financier notamment à travers le paiement mobile.

\section{LA METHODOLOGIE DE LA RECHERCHE}

Nous avons expliqué précédemment l'importance du mobile money en tant qu'accélérateur d'inclusion financière des populations exclues, il permet de lutter contre la pauvreté et réduire les disparités entre les genres. Les études menées par Bank Al Maghrib prouvent qu'il y a une forte disparité entre les régions, les milieux rural et urbain en matière d'inclusion financière.

L'objectif de cette étude empirique est de montrer que l'utilisation du mobile money permet de faciliter l'accès aux services financiers avant et pendant la crise sanitaire.

\section{A. Présentation des variables}

Pour mener notre analyse (l'utilisation de mobile money avant la crise et durant la crise et réduction d'inégalités), nous avons jugé utile d'utilisé deux types de variables :

Une variable dépendante, c'est la réduction d'inégalités qu'on va chercher à expliquer (variable dépendante) ;

Sept variables explicatives (indépendantes) de l'utilisation de mobile money :

- Le niveau d'étude

- Le genre

- Le lieu de résidence

- La catégorie socioprofessionnelle 
- Éducation financière

- L'âge

- Le revenu

\section{B. Les outils d'analyse}

L'analyse est effectuée par le logiciel SPSS 22 en utilisant les tests suivants :

- Le test d'inférence statistique du khi-deux qui permet de mesurer le degré d'association entre les variables qualitatives nominales et le coefficient de contingence Phi et Cramer qui permet d'évaluer l'intensité et la force de la relation.

- La Méthode ANOVA qui permet d'expliquer les variations de la variable dépendante par un seul facteur explicatif, développée par Fisher, l'analyse est complété par le coefficient de détermination R2 qui donne une idée sur l'existence d'une relation entre variables.

Le questionnaire était adressé à 250 personnes à travers plusieurs canaux (E-mail. Réseaux sociaux ...). Nous avons reçu 170 réponses composées de $58.8 \%$ d'hommes et $41.2 \%$ des femmes.

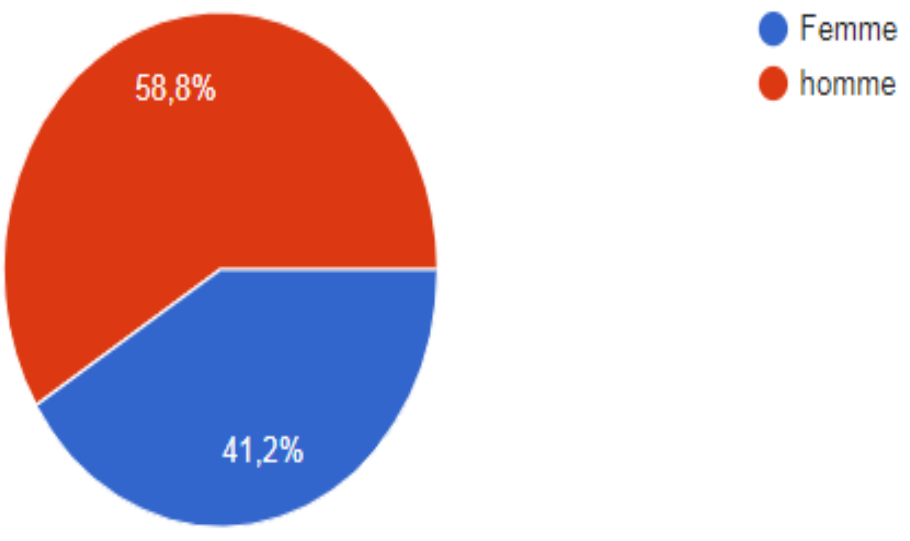

Figure 1. Répartition des répondants par sexe

156 personnes de l'échantillon ont un compte bancaire ou mobile soit $91.8 \%$ et 14 personnes n'ont pas un compte bancaire soit $8.2 \%$. Donc l'échantillon a estimé est constitué de 156 personnes (femmes et hommes).

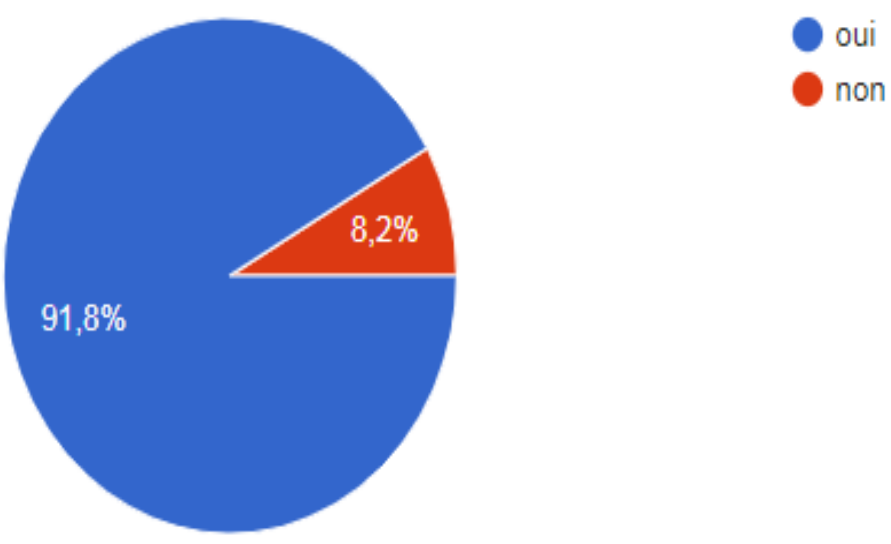

Figure 2.les personnes ayant un compte bancaire ou mobile 
Il s'agit d'un échantillon aléatoire simple. Les informations ont été recueillies par questionnaire

C. Les hypothèses à tester :

Tableau 1. Les hypothèses à tester

\begin{tabular}{|l|l|l|}
\hline \multicolumn{2}{|c|}{ Code } & \\
\hline \multirow{2}{*}{ H1 } & H1.1 & Le niveau d'étude à une influence sur l'utilisation du MM Avant la crise \\
\cline { 2 - 4 } & H1.2 & Le niveau d'étude à une influence sur l'utilisation du MM durant la crise \\
\hline \multirow{2}{*}{ H2 } & H2.1 & Le genre influence l'utilisation du MM avant la crise \\
\cline { 2 - 4 } & H2.2 & Le genre influence l'utilisation du MM durant la crise \\
\hline \multirow{2}{*}{ H3 } & H3.1 & Lieu de résidence influence l'utilisation du MM avant la crise \\
\cline { 2 - 4 } & H3.2 & Lieu de résidence influence l'utilisation du MM durant la crise \\
\hline H4 & H4.1 & La catégorie socioprofessionnelle influence l'utilisation du MM avant la crise \\
\cline { 2 - 4 } & H4.2 & La catégorie socioprofessionnelle influence l'utilisation du MM Durant la crise \\
\hline H5 & H5.1 & L'éducation financière influence l'utilisation du MM avant la crise \\
\cline { 2 - 4 } & H5.2 & L'éducation financière influence l'utilisation du MM durant la crise \\
\hline \multirow{2}{*}{ H6 } & H6.1 & L'âge influence l'utilisation du MM avant la crise \\
\cline { 2 - 4 } & H6.2 & L'âge influence l'utilisation du MM durant la crise \\
\hline \multirow{2}{*}{ H7 } & H7.1 & Le revenu influence l'utilisation du MM avant la crise \\
\cline { 2 - 4 } & H7.2 & Le revenu influence l'utilisation du MM durant la crise \\
\hline
\end{tabular}

\section{Le test des hypothèses}

La relation entre le niveau d'étude et l'utilisation du MM est analysée par le test de khi deux, les coefficients de contingence, Phi et Cramer nous permet de prononcer sur l'intensité de cette relation :

Test de l'hypothèse H1 (niveau d'étude)

$\mathrm{H} 0=$ aucune relation entre le niveau d'étude et l'utilisation du MM avant la crise

H1.1 = le niveau d'étude influence l'utilisation du MM avant la crise

Les résultats du test figurent dans le tableau suivant :

Tests du khi-deux

\begin{tabular}{|l|r|r|r|}
\hline & Valeur & ddl & Sig. approx. (bilatérale) \\
\hline khi-deux de Pearson & $2,655^{\mathrm{a}}$ & 5 &, 753 \\
Rapport de vraisemblance & 3,915 & 5 &, 562 \\
N d'observations valides & 156 & & \\
\hline
\end{tabular}

a. 8 cellules $(66,7 \%)$ ont un effectif théorique inférieur à 5. L'effectif théorique minimum est de ,28. 
Le test du Khi-deux nous permet de nous prononcer sur le lien entre le niveau d'étude et l'utilisation du MM. Il donne une probabilité de 0.75 qui est supérieure à 0.05 . De ce fait, on peut dire que le niveau d'étude n'a pas d'effet sur l'utilisation de MM avant la crise de notre échantillon.

L'intensité de la relation entre les deux variables est analysée par les coefficients de contingence, Phi et V de cramer. Les résultats du SPSS sont les suivants :

Tableau $\mathrm{N}^{\circ} 3$ : l'intensité de la relation entre le niveau d'étude et l'utilisation du MM avant la crise

Tableau 3. Mesures symétriques

\begin{tabular}{|ll|r|r|}
\hline & & Valeur & $\begin{array}{r}\text { Signification } \\
\text { approx. }\end{array}$ \\
\hline Nominal par Nominal & Phi &, 130 &, 753 \\
& V de Cramer &, 130 &, 753 \\
& Coefficient de contingence &, 129 &, 753 \\
N d'observations valides & & 156 & \\
\hline
\end{tabular}

Les statistiques Phi et V de Cramer ont une valeur de 0,130 sur une valeur maximale possible de 1. Cela représente une association très faible entre les deux variables (la relation est jugée très forte lorsque le coefficient V de cramer dépasse les 0.6). Quant au coefficient de contingence, sa valeur est de 0,129 qui est bien comprise entre 0 et 1 . Ceci traduit aussi une faible intensité de liaison entre les deux variables. De ce fait l'hypothèse H0 est acceptée et l'hypothèse H1.1 est rejetée : (Le Niveau d'étude n'a pas d'influence sur l'utilisation du MM Avant la crise.)

Tableau 2 : Appréciation de la relation entre le niveau d'étude et l'utilisation du MM Avant la crise

- $\quad$ Test de l'hypothèse H1.2

$\mathrm{H} 0=$ aucune relation entre le niveau d'étude et l'utilisation du MM durant la crise

H1.2 = le niveau d'étude influence l'utilisation du MM durant la crise

Les résultats du test figurent dans le tableau suivant :

Tableau 4 : Appréciation de la relation entre le niveau d'étude et l'utilisation du MM Durant la crise.

Tests du khi-deux

\begin{tabular}{|l|c|c|c|}
\hline & Valeur & ddl & $\begin{array}{c}\text { Sig. approx. } \\
\text { (bilatérale) }\end{array}$ \\
\hline khi-deux de Pearson & $3,977^{\mathrm{a}}$ & 10 &, 948 \\
Rapport de vraisemblance & 5,840 & 10 &, 828 \\
N d'observations valides & 156 & & \\
\hline
\end{tabular}

a. 13 cellules $(72,2 \%)$ ont un effectif théorique inférieur à 5 . L'effectif théorique minimum est de, 15

Le test du Khi-deux nous permet de nous prononcer sur le lien entre le niveau d'étude et l'utilisation du MM durant la crise. Il donne une probabilité de 0.948 qui est supérieure à 0.05 . De ce 
fait, on peut dire que le niveau d'étude n'a pas d'effet sur l'utilisation de MM durant la crise de notre échantillon.

L'intensité de la relation entre les deux variables est analysée par les coefficients de contingence, Phi et V de cramer. Les résultats du SPSS sont les suivants :

Tableau $\mathrm{N}^{\circ} 5$ : l'intensité de la relation entre le niveau d'étude et l'utilisation du MM durant la crise

\begin{tabular}{|ll|r|r|}
\hline \multicolumn{2}{|c|}{ Mesures symétriques } & \multicolumn{2}{c|}{$\begin{array}{c}\text { Signification } \\
\text { approx. }\end{array}$} \\
\hline Nominal par Nominal & Phi & Valeur &, 160 \\
& V de Cramer &, 113 &, 948 \\
& Coefficient de contingence &, 158 &, 948 \\
N d'observations valides & & 156 & \\
\hline
\end{tabular}

Les statistiques Phi et V de Cramer ont une valeur de 0,160 sur une valeur maximale possible de 1 . Cela représente une association très faible entre les deux variables (la relation est jugée très forte lorsque le coefficient V de cramer dépasse les 0.6). Quant au coefficient de contingence, sa valeur est de 0,160 elle est bien comprise entre 0 et 1 . Ceci traduit aussi une faible intensité de liaison entre les deux variables. De ce fait l'hypothèse H0 est acceptée et l'hypothèse H1.2 est rejetée : (Le Niveau d'étude n'a pas d'influence sur l'utilisation du MM Durant la crise.)

Test de l'hypothèse H2 (le genre)

$\mathrm{H} 0=$ aucune relation entre le genre et l'utilisation du MM avant la crise

$\mathrm{H} 2.1$ = le genre influence l'utilisation du MM avant la crise

- $\quad$ Test de l'hypothèse H2.1

Les résultats du test figurent dans le tableau suivant :

Tableau 6 : Appréciation de la relation entre le genre et l'utilisation du MM Avant la crise.

Tests du khi-deux

\begin{tabular}{|l|r|r|r|r|r|}
\hline & Valeur & ddl & $\begin{array}{c}\text { Sig. approx. } \\
\text { (bilatérale) }\end{array}$ & $\begin{array}{c}\text { Sig. exacte } \\
\text { (bilatérale) }\end{array}$ & $\begin{array}{c}\text { Sig. exacte } \\
\text { (unilatérale) }\end{array}$ \\
\hline khi-deux de Pearson & $7,552^{\mathrm{a}}$ & 1 &, 006 & & \\
Correction pour continuitéb & 6,574 & 1 &, 010 &, 009 & \\
Rapport de vraisemblance & 7,421 & 1 &, 006 & & \\
Test exact de Fisher & & & & & \\
N d'observations valides & 156 & & & & \\
\hline
\end{tabular}

a. 0 cellules $(0,0 \%)$ ont un effectif théorique inférieur à 5 . L'effectif théorique minimum est de 16,54.

b. Calculée uniquement pour une table $2 \times 2$ 
Le test du Khi-deux nous permet de nous prononcer sur le lien entre le genre et l'utilisation du MM avant la crise. Il donne une probabilité de 0.006 qui est inférieure à 0.05 . De ce fait, on peut dire que le genre a un effet sur l'utilisation de MM avant la crise de notre échantillon.

L'intensité de la relation entre les deux variables est analysée par les coefficients de contingence, Phi et V de cramer. Les résultats du SPSS sont les suivants :

Tableau 7. L'intensité de la relation entre le genre et l'utilisation du MM avant la crise

\begin{tabular}{|ll|r|r|}
\hline \multicolumn{2}{|c|}{ Mesures symétriques } & \multicolumn{2}{c|}{$\begin{array}{c}\text { Signification } \\
\text { approx. }\end{array}$} \\
\hline Nominal par Nominal & Phi & Valeur &, 220 \\
& V de Cramer &, 220 &, 006 \\
& Coefficient de contingence &, 215 &, 006 \\
& & 156 & \\
N d'observations valides & &
\end{tabular}

Les statistiques Phi et V de Cramer ont une valeur de 0,220 sur une valeur maximale possible de 1. Cela représente une relation moyenne entre les deux variables (la relation est jugée très forte lorsque le coefficient V de cramer dépasse les 0.6). Quant au coefficient de contingence, sa valeur est de 0,215 elle est bien comprise entre 0 et 1 . Ceci traduit aussi une intensité moyenne de liaison entre les deux variables.

De ce fait l'hypothèse $\mathrm{H} 0$ est rejetée et l'hypothèse H2.1 est acceptée : (Le genre a une influence sur l'utilisation du MM Avant la crise.)

- $\quad$ Test de l'hypothèse H2.2

$\mathrm{H} 0$ = aucune relation entre le genre et l'utilisation du MM durant la crise

H2.2 = le genre influence l'utilisation du MM durant la crise

Tableau 8. Appréciation de la relation entre le genre et l'utilisation du MM durant la crise.

Tests du khi-deux

\begin{tabular}{|l|r|r|r|}
\hline & Valeur & ddl & $\begin{array}{c}\text { Sig. approx. } \\
\text { (bilatérale) }\end{array}$ \\
\hline khi-deux de Pearson & $8,162^{\mathrm{a}}$ & 2 & 017 \\
Rapport de vraisemblance & 7,939 & 2 &, 019 \\
N d'observations valides & 156 & & \\
\hline
\end{tabular}

a. 0 cellules $(0,0 \%)$ ont un effectif théorique inférieur à 5 . L'effectif théorique minimum est de 8,85 .

Le test du Khi-deux nous permet de nous prononcer sur le lien entre le genre et l'utilisation du MM durant la crise. Il donne une probabilité de 0.017 qui est inférieure à 0.05 . De ce fait, on peut dire que le genre a un effet sur l'utilisation de MM durant la crise de notre échantillon. 
L'intensité de la relation entre les deux variables est analysée par les coefficients de contingence, Phi et V de cramer. Les résultats du SPSS sont les suivants :

Tableau 9. L'intensité de la relation entre le genre et l'utilisation du MM durant la crise

\begin{tabular}{|ll|r|r|}
\hline \multicolumn{2}{|c|}{ Mesures symétriques } & Signification \\
approx.
\end{tabular}

Les statistiques Phi et V de Cramer ont une valeur de 0,3 sur une valeur maximale possible de 1. Cela représente une relation moyenne entre les deux variables (la relation est jugée très forte lorsque le coefficient $\mathrm{V}$ de cramer dépasse les 0.6). Quant au coefficient de contingence, sa valeur est de 0,223 elle est bien comprise entre 0 et 1 . Ceci traduit aussi une intensité de liaison moyenne entre les deux variables.

De ce fait l'hypothèse $\mathrm{H} 0$ est rejetée et l'hypothèse H2.2 est acceptée : (Le genre a une influence sur l'utilisation du MM durant la crise.)

Test de l'hypothèse H3 (le milieu de résidence)

H0 = aucune relation entre milieu de résidence et l'utilisation du MM avant la crise

H3.1 = le milieu de résidence influence l'utilisation du MM avant la crise

Tableau 10 : Appréciation de la relation entre le milieu de résidence et l'utilisation du MM avant la crise

Tests du khi-deux

\begin{tabular}{|l|r|r|r|r|r|}
\hline & Valeur & ddl & Sig. approx. (bilatérale) & Sig. exacte (bilatérale) & Sig. exacte (unilatérale) \\
\hline khi-deux de Pearson &, $028^{\mathrm{a}}$ & 1 &, 868 & & \\
Correction pour continuitéb &, 000 & 1 & 1,000 & & \\
Rapport de vraisemblance &, 028 & 1 &, 866 & & \\
Test exact de Fisher & & & & & \\
N d'observations valides & 156 & & & & \\
\hline
\end{tabular}

a. 1 cellules $(25,0 \%)$ ont un effectif théorique inférieur à 5. L'effectif théorique minimum est de 2,21.

b. Calculée uniquement pour une table $2 \times 2$

Le test du Khi-deux nous permet de nous prononcer sur le lien entre le milieu de résidence et l'utilisation du MM avant la crise. Il donne une probabilité de 0.868 qui est supérieure à 0.05 . De ce fait, on peut dire que le milieu de résidence n'a aucun effet sur l'utilisation de MM avant la crise de notre échantillon. 
L'intensité de la relation entre les deux variables est analysée par les coefficients de contingence, Phi et V de cramer. Les résultats du SPSS sont les suivants :

Tableau 11 : l'intensité de la relation entre le milieu de résidence et l'utilisation du MM avant la crise

\begin{tabular}{|ll|r|r|}
\hline \multicolumn{2}{|c|}{ Mesures symétriques } & \multicolumn{2}{c|}{$\begin{array}{c}\text { Signification } \\
\text { approx. }\end{array}$} \\
\hline Nominal par Nominal & Phi & Valeur &, 013 \\
& V de Cramer &, 013 &, 868 \\
& Coefficient de contingence &, 013 &, 868 \\
& & 156 & \\
N d'observations valides & &
\end{tabular}

Les statistiques Phi et V de Cramer ont une valeur de 0,13 sur une valeur maximale possible de 1 . Cela représente une relation faible entre les deux variables (la relation est jugée très forte lorsque le coefficient V de cramer dépasse les 0.6). Quant au coefficient de contingence, sa valeur est de 0,013 elle est bien comprise entre 0 et 1 . Ceci traduit aussi une intensité de liaison faible entre les deux variables.

De ce fait l'hypothèse $\mathrm{H} 0$ est acceptée et l'hypothèse H3.1 est rejetée : (Le milieu de résidence n'a pas d'influence sur l'utilisation du MM Avant la crise.)

- $\quad$ Test de l'hypothèse H3.2

H0 = aucune relation entre le milieu de résidence et l'utilisation du MM durant la crise

H3.1 = la location influence l'utilisation du MM durant la crise

Tableau 12 : Appréciation de la relation entre la location et l'utilisation du MM durant la crise

\begin{tabular}{|l|r|r|r|}
\hline \multicolumn{1}{|l|}{ Tests du khi-deux } \\
& Valeur & ddl & $\begin{array}{c}\text { Sig. approx. } \\
\text { (bilatérale) }\end{array}$ \\
\hline khi-deux de Pearson & $1,005^{\mathrm{a}}$ & 2 &, 605 \\
Rapport de vraisemblance & 1,120 & & 2 \\
N d'observations valides & 156 & &, 571 \\
\hline
\end{tabular}

a. 3 cellules $(50,0 \%)$ ont un effectif théorique inférieur à 5 . L'effectif théorique minimum est de 1,18 .

Le test du Khi-deux nous permet de nous prononcer sur le lien entre la location et l'utilisation du MM durant la crise. Il donne une probabilité de 0.605 qui est supérieure à 0.05 . De ce fait, on peut dire que la location n'a pas d'effet sur l'utilisation de MM durant la crise de notre échantillon.

L'intensité de la relation entre les deux variables est analysée par les coefficients de contingence, Phi et V de cramer. Les résultats du SPSS sont les suivants : 
Tableau 13 : l'intensité de la relation entre le milieu de résidence et l'utilisation du MM durant la crise

\begin{tabular}{|ll|r|r|}
\hline \multicolumn{2}{|c|}{ Mesures symétriques } \\
\hline Nominal par Nominal & Phi & Valeur & Signification approx. \\
& V de Cramer &, 080 &, 605 \\
& Coefficient de contingence &, 080 &, 605 \\
& &, 080 &, 605 \\
N d'observations valides & & 156 & \\
\hline
\end{tabular}

Les statistiques Phi et V de Cramer ont une valeur de 0,080 sur une valeur maximale possible de 1. Cela représente une relation faible entre les deux variables (la relation est jugée très forte lorsque le coefficient $\mathrm{V}$ de cramer dépasse les 0.6). Quant au coefficient de contingence, sa valeur est de 0,080 elle est bien comprise entre 0 et 1 . Ceci traduit aussi une intensité de liaison faible entre les deux variables.

De ce fait l'hypothèse $\mathrm{H} 0$ est acceptée et l'hypothèse H3.2 est rejetée : (le milieu de résidence n'a pas d'influence sur l'utilisation du MM durant la crise.)

\section{Test de l'hypothèse H4 (catégorie socioprofessionnelle)}

Test de l'hypothèse H4.1

$\mathrm{H} 0$ = aucune relation entre CS et l'utilisation du MM Avant la crise

H4.1 = la CS influence l'utilisation du MM avant la crise

Tableau 14 : Appréciation de la relation entre CS et l'utilisation du MM avant la crise

Tests du khi-deux

\begin{tabular}{|l|c|c|c|}
\hline & Valeur & ddl & $\begin{array}{c}\text { Sig. approx. } \\
\text { (bilatérale) }\end{array}$ \\
\hline khi-deux de Pearson & $4,795^{\mathrm{a}}$ & 10 &, 904 \\
Rapport de vraisemblance & 4,949 & 10 &, 895 \\
N d'observations valides & 156 & & \\
\hline
\end{tabular}

a. 15 cellules $(68,2 \%)$ ont un effectif théorique inférieur à 5 . L'effectif théorique minimum est de 28 .

Le test du Khi-deux nous permet de nous prononcer sur le lien entre la CS et l'utilisation du MM avant la crise. Il donne une probabilité de 0.904 qui est supérieure à 0.05 . De ce fait, on peut dire que la location n'a pas d'effet sur l'utilisation de MM avant la crise de notre échantillon.

L'intensité de la relation entre les deux variables est analysée par les coefficients de contingence, Phi et V de cramer. Les résultats du SPSS sont les suivants : 
Tableau 15 : l'intensité de la relation entre CS et l'utilisation du MM avant la crise

\begin{tabular}{|ll|r|r|}
\hline \multicolumn{2}{|c|}{ Mesures symétriques } & Signification \\
& & Valeur & approx. \\
\hline Nominal par Nominal & Phi &, 175 &, 904 \\
& V de Cramer &, 175 &, 904 \\
& Coefficient de contingence &, 173 &, 904 \\
\hline N d'observations valides & & 156 & \\
\hline
\end{tabular}

Les statistiques Phi et V de Cramer ont une valeur de 0,175 sur une valeur maximale possible de 1. Cela représente une relation faible entre les deux variables (la relation est jugée très forte lorsque le coefficient $\mathrm{V}$ de cramer dépasse les 0.6). Quant au coefficient de contingence, sa valeur est de 0,173 qui est bien comprise entre 0 et 1 . Ceci traduit aussi une intensité de liaison faible entre les deux variables.

De ce fait l'hypothèse $\mathrm{H} 0$ est acceptée et l'hypothèse H4.1. est rejetée : (La CS n'a pas d'influence sur l'utilisation du MM Avant la crise.)

- $\quad$ Test de l'hypothèse H4.2

$\mathrm{H} 0$ = aucune relation entre CS et l'utilisation du MM durant la crise

H4.2 = la CS influence l'utilisation du MM durant la crise

Tableau 16 : Appréciation de la relation entre CS et l'utilisation du MM durant la crise

Tests du khi-deux

\begin{tabular}{|l|c|c|c|}
\hline & Valeur & ddl & $\begin{array}{c}\text { Sig. approx. } \\
\text { (bilatérale) }\end{array}$ \\
\hline khi-deux de Pearson & $22,470^{\mathrm{a}}$ & 20 &, 316 \\
Rapport de vraisemblance & 25,035 & 20 &, 200 \\
N d'observations valides & 156 & & \\
\hline
\end{tabular}

a. 25 cellules $(75,8 \%)$ ont un effectif théorique inférieur à 5 . L'effectif théorique minimum est de, 15 .

Le test du Khi-deux nous permet de nous prononcer sur le lien entre la CS et l'utilisation du MM durant la crise. Il donne une probabilité de 0.316 qui est supérieure à 0.05 . De ce fait, on peut dire que la location n'a pas d'effet sur l'utilisation de MM avant la crise de notre échantillon.

L'intensité de la relation entre les deux variables est analysée par les coefficients de contingence, Phi et V de cramer. Les résultats du SPSS sont les suivants : 
Tableau 17 : l'intensité de la relation entre CS et l'utilisation du MM durant la crise

\begin{tabular}{|c|c|c|c|}
\hline \multicolumn{4}{|c|}{ Mesures symétriques } \\
\hline & & Valeur & $\begin{array}{c}\text { Signification } \\
\text { approx. }\end{array}$ \\
\hline \multirow[t]{3}{*}{ Nominal par Nominal } & Phi & 380 & ,316 \\
\hline & V de Cramer & ,268 & ,316 \\
\hline & Coefficient de contingence & ,355 &, 316 \\
\hline $\mathrm{N}$ d'observations valide & & 156 & \\
\hline
\end{tabular}

Les statistiques Phi et V de Cramer ont une valeur de 0,380 sur une valeur maximale possible de 1. Cela représente une relation faible entre les deux variables (la relation est jugée très forte lorsque le coefficient $\mathrm{V}$ de cramer dépasse les 0.6). Quant au coefficient de contingence, sa valeur est de 0,355 qui est bien comprise entre 0 et 1 . Ceci traduit aussi une intensité de liaison faible entre les deux variables.

De ce fait l'hypothèse $\mathrm{H} 0$ est acceptée et l'hypothèse H4.2. est rejetée : (La CS n'a pas d'influence sur l'utilisation du MM durant la crise.)

Test de l'hypothèse H5 (Éducation financière)

- $\quad$ Test de l'hypothèse H5.1

$\mathrm{H} 0$ = aucune relation entre EF et l'utilisation du MM Avant la crise

H5.1 = EF influence l'utilisation du MM Avant la crise

Tableau 18 : l'intensité de la relation entre EF et l'utilisation du MM Avant la crise

\begin{tabular}{|ll|r|r|}
\hline \multicolumn{2}{|c|}{ Mesures symétriques } & Signification \\
& & Valeur & approx. \\
\hline Nominal par Nominal & Phi &, 027 &, 732 \\
& V de Cramer &, 027 &, 732 \\
& Coefficient de contingence &, 027 &, 732 \\
\hline N d'observations valides & & 156 & \\
\hline
\end{tabular}

Les statistiques Phi et V de Cramer ont une valeur de 0,027 sur une valeur maximale possible de 1. Cela représente une relation faible entre les deux variables (la relation est jugée très forte lorsque le coefficient $\mathrm{V}$ de cramer dépasse les 0.6). Quant au coefficient de contingence, sa valeur est de 0,027 qui est bien comprise entre 0 et 1 . Ceci traduit aussi une intensité de liaison faible entre les deux variables. De ce fait l'hypothèse H0 
est acceptée et l'hypothèse H5.1. est rejetée : (L'éducation financière n'a pas d'influence sur l'utilisation du MM Avant la crise.)

- $\quad$ Test de l'hypothèse H5.2

H0 = aucune relation entre EF et l'utilisation du MM Durant la crise

H5.1 = EF influence l'utilisation du MM Durant la crise

Tableau 19: Appréciation de la relation entre EF et l'utilisation du MM Durant la crise

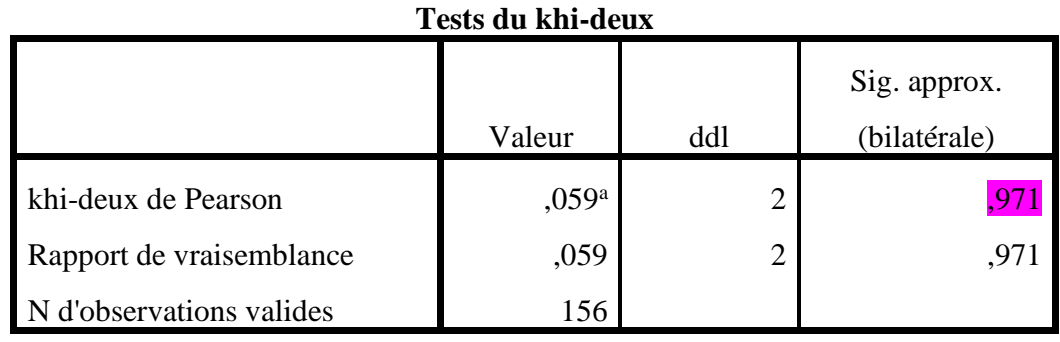

a. 0 cellules $(0,0 \%)$ ont un effectif théorique inférieur à 5 . L'effectif théorique

minimum est de 6,34 .

Le test du Khi-deux nous permet de nous prononcer sur le lien entre l'EF et l'utilisation du MM Durant la crise. Il donne une probabilité de 0.971 qui est supérieure à 0.05 . De ce fait, on peut dire que l'EF n'a pas d'effet sur l'utilisation de MM durant la crise de notre échantillon.

L'intensité de la relation entre les deux variables est analysée par les coefficients de contingence, Phi et V de cramer. Les résultats du SPSS sont les suivants:

Tableau 20 : l'intensité de la relation entre EF et l'utilisation du MM Durant la crise

\begin{tabular}{|c|c|c|}
\hline \multicolumn{3}{|l|}{ Mesures symétriques } \\
\hline & Valeur & Signification approx. \\
\hline Nominal par Nominal Phi &, 019 & ,971 \\
\hline V de Cramer & 019 & ,971 \\
\hline Coefficient de contingence &, 019 & ,971 \\
\hline $\mathrm{N}$ d'observations valides & 156 & \\
\hline
\end{tabular}

Les statistiques Phi et V de Cramer ont une valeur de 0,019 sur une valeur maximale possible de 1. Cela représente une relation faible entre les deux variables (la relation est jugée très forte lorsque le coefficient $\mathrm{V}$ de cramer dépasse les 0.6). Quant au coefficient de contingence, sa valeur est de 0,019 qui est bien comprise entre 0 et 1 . Ceci traduit aussi une intensité de liaison faible entre les deux variables. 
De ce fait l'hypothèse $\mathrm{H} 0$ est acceptée et l'hypothèse H5.2. est rejetée : (L'éducation financière n'a pas d'influence sur l'utilisation du MM durant la crise.)

\section{Test de l'hypothèse H6 (l'âge)}

- $\quad$ Test de l'hypothèse H6.1

H0 = aucune relation entre l'âge et l'utilisation du MM Avant la crise

H6.1 = l'âge influence l'utilisation du MM Avant la crise

Tableau Nº 21 : Résultats de l'ANOVA

ANOVA $^{\mathrm{a}}$

\begin{tabular}{|ll|r|r|r|r|r|}
\hline \multicolumn{2}{|l|}{ Modèle } & Somme des carrés & ddl & Carré moyen & F & Sig. \\
\hline 1 & Régression & 1,019 & 1 & 1,019 &, 860 &, $355^{\text {b }}$ \\
& Résidus & 182,538 & 154 & 1,185 & & \\
Total & 183,558 & 155 & & & \\
\hline
\end{tabular}

a. Variable dépendante : L'âge

b. Prédicteurs : (Constante), PM avant la crise

Tableau $\mathrm{N}^{\circ} 22$ : Coefficients du modèle

\begin{tabular}{|c|c|c|c|c|c|}
\hline \multicolumn{6}{|c|}{ Coefficients $^{\mathrm{a}}$} \\
\hline \multirow[b]{2}{*}{ Modèle } & \multicolumn{2}{|c|}{ Coefficients non standardisés } & \multirow{2}{*}{\begin{tabular}{|c|} 
Coefficients standardisés \\
Bêta \\
\end{tabular}} & \multirow[b]{2}{*}{$\mathrm{t}$} & \multirow[b]{2}{*}{ Sig. } \\
\hline & $\mathrm{B}$ & Ecart standard & & & \\
\hline 1 (Constante) & 2,173 & ,264 & & 8,242 &, 000 \\
\hline PM avant la crise &, 181 & ,195 &, 075 & ,927 & ,355 \\
\hline
\end{tabular}

a. Variable dépendante : L'âge

Le résultat obtenu nous permet de nous prononcer sur le lien entre l'âge et l'utilisation du MM avant la crise. L'ANOVA donne un niveau de signification 0.355 qui est supérieure à 0.05 donc on peut dire que le modèle est globalement significatif au seuil de $10 \%$. La variable (âge) a une influence faible sur l'utilisation du MM AVANT la crise.

Ce résultat est confirmé par le coefficient de détermination R2 qui tend vers $0(0.075)$ c'est-à-dire la relation fonctionnelle entre les deux variables est relativement faible.

De ce fait l'hypothèse H0 est rejetée et l'hypothèse H6.1. est acceptée (L'âge a une influence sur l'utilisation du MM Avant la crise.)

- $\quad$ Test de l'hypothèse H6.2

H0 = aucune relation entre l'âge et l'utilisation du MM durant la crise

H6.1 = l'âge influence l'utilisation du MM durant la crise 
Tableau $\mathrm{N}^{\circ} 23$ : Résultats de l'ANOVA

\begin{tabular}{|l|r|r|r|r|r|}
\hline Modèle & Somme des carrés & ddl & Carré moyen & F & Sig. \\
\hline 1 Régression &, 251 & 1 &, 251 &, 211 &, $647^{\mathrm{b}}$ \\
Résidus & 183,307 & 154 & 1,190 & & \\
Total & 183,558 & 155 & & & \\
\hline
\end{tabular}

a. Variable dépendante : L'âge

b. Prédicteurs : (Constante), PM durant la crise

Tableau $\mathrm{N}^{\circ} 24$ : Coefficients du modèle

\begin{tabular}{|c|c|c|c|c|c|}
\hline \multirow[b]{3}{*}{ Modèle } & \multicolumn{3}{|c|}{ Coefficients $^{\mathrm{a}}$} & \multirow[b]{3}{*}{$\mathrm{t}$} & \multirow[b]{3}{*}{ Sig. } \\
\hline & \multicolumn{2}{|c|}{ Coefficients non standardisés } & Coefficients standardisés & & \\
\hline & $\mathrm{B}$ & Ecart standard & Bêta & & \\
\hline 1 (Constante) & 2,296 & ,251 & & 9,144 & ,000 \\
\hline PM durant la crise & ,046 & 101 & 037 & ,459 & ,647 \\
\hline
\end{tabular}

a. Variable dépendante : L'âge

Le résultat obtenu nous permet de nous prononcer sur le lien entre l'âge et l'utilisation du MM durant la crise.

L'ANOVA donne un niveau de signification 0.647 qui est supérieure à 0.05 donc on peut dire que le modèle est globalement significatif au seuil de $10 \%$. La variable (âge) a une influence faible sur l'utilisation du MM durant la crise au sein des coopératives de l'échantillon.

Ce résultat est confirmé par le coefficient de détermination R2 qui tend vers $0(0.037)$ c'est-à-dire la relation fonctionnelle entre les deux variables est relativement faible.

De ce fait l'hypothèse $\mathrm{H} 0$ est rejetée et l'hypothèse H6.2. est acceptée (L'âge a une influence sur l'utilisation du MM durant la crise.)

Test de l'hypothèse H7 (le revenu)

- $\quad$ Test de l'hypothèse H7.1

H0 = aucune relation entre le revenu et l'utilisation du MM avant la crise

H7.1 = le revenu influence l'utilisation du MM avant la crise 
Tableau $\mathrm{N}^{\circ} 25$ : Résultats de l'ANOVA

\begin{tabular}{|c|c|c|c|c|c|c|}
\hline \multicolumn{7}{|c|}{ ANOVA $^{a}$} \\
\hline & & Somme des carrés & ddl & Carré moyen & $\mathrm{F}$ & Sig. \\
\hline \multirow[t]{3}{*}{1} & Régression &, 060 & 1 &, 060 & ,076 &, $783^{b}$ \\
\hline & Résidus & 122,529 & 154 & ,796 & & \\
\hline & Total & 122,590 & 155 & & & \\
\hline
\end{tabular}

a. Variable dépendante : Revenu

b. Prédicteurs : (Constante), PM avant la crise

Tableau $\mathrm{N}^{\circ} 26$ : Coefficients du modèle

\begin{tabular}{|c|c|c|c|c|c|}
\hline \multicolumn{6}{|c|}{ Coefficients $^{\mathrm{a}}$} \\
\hline \multirow[b]{2}{*}{ Modèle } & \multicolumn{2}{|c|}{ Coefficients non standardisés } & \multirow{2}{*}{$\begin{array}{c}\text { Coefficients standardisés } \\
\text { Bêta }\end{array}$} & \multirow[b]{2}{*}{$\mathrm{t}$} & \multirow[b]{2}{*}{ Sig. } \\
\hline & $\mathrm{B}$ & Ecart standard & & & \\
\hline 1 (Constante) & 1,726 &, 216 & & 7,989 &, 000 \\
\hline PM avant la crise &, 044 &, 160 &, 022 & ,276 &, 783 \\
\hline
\end{tabular}

a. Variable dépendante : Revenu

Le résultat obtenu nous permet de nous prononcer sur le lien entre le revenu et l'utilisation du MM avant la crise. L'ANOVA donne un niveau de signification 0.783 qui est supérieure à 0.05 donc on peut dire que le modèle est globalement significatif au seuil de $10 \%$. La variable (revenu) a une influence faible sur l'utilisation du MM avant la crise.

Ce résultat est confirmé par le coefficient de détermination R2 qui tend vers $0(0.022)$ c'est-à-dire la relation fonctionnelle entre les deux variables est relativement faible.

De ce fait l'hypothèse $\mathrm{H} 0$ est rejetée et l'hypothèse H7.1. est acceptée (Le revenu a une influence sur l'utilisation du MM Avant la crise.)

- $\quad$ Test de l'hypothèse H7.2

H0 = aucune relation entre le revenu et l'utilisation du MM durant la crise

H7.1 = le revenu influence l'utilisation du MM durant la crise

Tableau $\mathrm{N}^{\circ} 27$ : Résultats de l’ANOVA

\begin{tabular}{|ll|r|r|r|r|r|}
\hline \multicolumn{1}{|l|}{} & ANOVÀ̀le & & \\
\hline 1 & Somme des carrés & ddl & Carré moyen & F & Sig. \\
\hline & Régression & 4,204 & 1 & 4,204 & 5,469 &, $021^{\mathrm{b}}$ \\
& Résidus & 118,385 & 154 &, 769 & & \\
Total & 122,590 & 155 & & & \\
\hline
\end{tabular}

a. Variable dépendante : Revenu

b. Prédicteurs : (Constante), PM durant la crise 
Tableau $\mathrm{N}^{\circ} 28$ : Coefficients du modèle

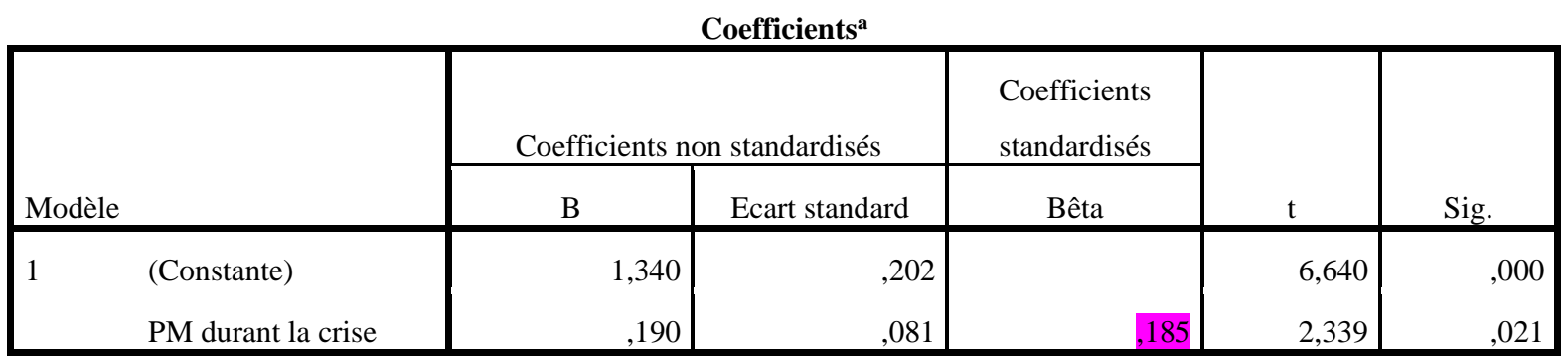

a. Variable dépendante : Revenu

Le résultat obtenu nous permet de nous prononcer sur le lien entre le revenu et l'utilisation du MM durant la crise. L'ANOVA donne un niveau de signification 0.021 qui est inférieure à 0.05 donc on peut dire que le modèle est globalement significatif au seuil de 10\%. La variable (revenu) a une influence forte sur l'utilisation du MM durant la crise au sein des coopératives de l'échantillon.

Ce résultat est confirmé par le coefficient de détermination R2 qui tend vers 1 (0.185) c'est-à-dire la relation fonctionnelle entre les deux variables est relativement forte.

De ce fait l'hypothèse $\mathrm{H} 0$ est rejetée et l'hypothèse H7.1 est acceptée (Le revenu a une influence sur l'utilisation du MM durant la crise.)

\section{RESUlTATS ET DISCUSSION}

Dans notre étude nous avons analysé la relation entre les variables qui peuvent influencer l'utilisation de mobile money ou le paiement mobile sur deux périodes: avant la crise et durant la crise, sur la réduction d'inégalités d'accès aux services financiers.

A. Les résultats des estimations des variables avant la crise :

Après l'estimation des variables par le logiciel SPSS 22, nous avons obtenu les résultats suivants :

\begin{tabular}{|c|l|l|l|}
\hline \multirow{5}{*}{$\begin{array}{c}\text { Estimation des } \\
\text { variables avant la crise }\end{array}$} & \multicolumn{1}{|c|}{ Les variables } & \multicolumn{1}{c|}{ Test Khi2 } & \multicolumn{1}{c|}{ V /phi de cramer } \\
\cline { 2 - 4 } & Le niveau d'étude & Non significative & Faible influence \\
\cline { 2 - 4 } & Le genre & significative & Moyenne influence \\
\cline { 2 - 4 } & Le milieu de résidence & Non significative & Faible influence \\
\cline { 2 - 5 } & $\begin{array}{l}\text { La Catégorie } \\
\text { socioprofessionnelle }\end{array}$ & Non significative & Faible influence \\
\cline { 2 - 5 } & L'éducation financière & Non significative & Faible influence \\
\hline
\end{tabular}

Le test de Khi-2 indique la significativité de la variable

-V/phi de cramer explique la force de relation entre les variables

\begin{tabular}{|l|l|l|l|}
\hline \multirow{3}{*}{ Estimation avant la crise } & \multicolumn{1}{|c|}{ Les variables } & \multicolumn{1}{c|}{ ANOVA } & \multicolumn{1}{c|}{$\begin{array}{c}\text { Le coefficient de } \\
\text { détermination } \mathrm{R}\end{array}$} \\
\cline { 2 - 4 } & \multicolumn{1}{|c|}{ L'âge } & Non significative & Faible relation \\
\cline { 2 - 4 } & Le revenu & Non significative & Faible relation \\
\hline
\end{tabular}


D'après les résultats suivants, on peut dire que tous les variables ont une relation non significative ( $\mathrm{p}$ moins de 0.05), ce qui était confirmer par les tests de la force de relation (V/phi de cramer) (coefficient de détermination R) qui a indiqué que la force de relation est faible, sauf la variable Genre qui a une influence sur l'utilisation de mobile money avec une influence moyenne.

\section{Les résultats des estimations des variables durant la crise :}

Après l'estimation des variables par le logiciel SPSS 22, nous avons obtenu les résultats suivants :

\begin{tabular}{|l|l|l|l|}
\hline \multirow{5}{*}{$\begin{array}{l}\text { Estimation des variables } \\
\text { durant la crise }\end{array}$} & Les variables & Test Khi2 & V /phi de cramer \\
\cline { 2 - 4 } & Le niveau d'étude & Non significative & Faible influence \\
\cline { 2 - 4 } & Le genre & significative & Moyenne influence \\
\cline { 2 - 4 } & Le milieu de résidence & Non significative & Faible influence \\
\cline { 2 - 4 } & $\begin{array}{l}\text { La Catégorie } \\
\text { socioprofessionnelle }\end{array}$ & Non significative & Faible influence \\
\cline { 2 - 4 } & L'éducation financière & Non significative & Faible influence \\
\hline
\end{tabular}

\begin{tabular}{|c|c|c|c|}
\hline \multirow{3}{*}{ Estimation durant la crise } & Les variables & ANOVA & $\begin{array}{c}\text { Le coefficient de } \\
\text { détermination } \mathrm{R}\end{array}$ \\
\cline { 2 - 4 } & L'âge & Non significative & Faible relation \\
\cline { 2 - 4 } & Le revenu & significative & Forte relation \\
\hline
\end{tabular}

Donc d'après les résultats d'estimation, nous pouvons conclure que deux variables influencent l'utilisation de mobile money durant la crise covis-19, notamment le genre qui a une relation moyenne et le revenu qui a une forte relation sur l'utilisation de mobile money et donc les réductions d'inégalités.

D'après les résultats inscrits dans la partie d'estimation, l'augmentation du niveau d'étude, le milieu de résidence, la catégorie socioprofessionnelle, l'éducation financière et l'âge n'ont pas une influence sur l'augmentation d'utilisation de mobile money avant la crise, donc nous avons accepté l'hypothèse H0 et nous avons rejeté l'hypothèse $\mathrm{H} 1$.

Par contre le genre a une influence sur l'utilisation de mobile money avant la crise. Donc l'hypothèse H1 a été acceptée.

Deux variables à savoir : le genre et le revenu, augmentent significativement l'utilisation de mobile money durant la crise et réduit l'inégalité d'accès aux services financiers ce qui est expliquer par la réponse des interrogés, que le manque d'argent est une cause principale de ne pas avoir un compte en banque ou compte de paiement mobile .en effet il existe une relation entre le revenu et la réduction d'inégalités. 


\section{B. Discussion des résultats}

L'utilisation de paiement mobile est un levier d'inclusion financière et réductions d'inégalités des revenus et d'accès aux services financiers. Le contexte de la crise covid-19 a accéléré la digitalisation des services financiers, dans ce sens les résultats de cette étude présente qu'il y a une relation significative entre le genre et l'utilisation de mobile money (58.8\% hommes, $41.2 \%$ femmes sur un échantillon de 170 personnes). En effet, les femmes n'ont pas suffisamment d'opportunités d'utilisation de paiements mobiles pour plusieurs raisons : absence de flux de trésorerie réguliers, elles ne savent pas comment utiliser les applications sur les smartphones, le refus de commerçants dans les zones rurales de paiement mobile. Ziadi, (2013) souligne que dans les pays à revenu moyen, l'exclusion financière touche principalement les femmes, les personnes ayant de faibles revenus, les moins instruits et celles du milieu rural.

En ce qui concerne la relation entre le revenu et réductions d'inégalités, les études de Demir et al (2020) ont montré que Le mobile money favorise l'inclusion financière et réduit significativement les inégalités de revenus sur 140 pays. Dans notre étude, nous avons 42.4\% des interrogés (170 personnes dans l'échantillon) ayant un revenu élevé (plus de $7000 \mathrm{dh}$ ), ce qui confirme la forte relation entre le niveau de revenu et l'utilisation de paiement mobile.

La stratégie nationale d'inclusion financière 2019 considère l'éducation financière comme une composante importante pour soutenir l'utilisation de mobile money, puisque 50\% de notre échantillon n'ont jamais entendu parler de paiement mobile et $73.5 \%$ ont besoin d'éducation financière ce qui est confirmé par la préférence de la monnaie liquide. Parmi les freins au recourt au paiement mobile on trouve :

-Une faible éducation financière

-Absence de provision

-Le manque de confiance à ce genre de paiement

- Des problèmes techniques liés à l'instabilité du réseau

Donc l'éducation financière est un levier important pour favoriser la généralisation de l'utilisation du paiement mobile et la réduction des inégalités entre les populations défavorisées principalement les femmes et les jeunes.

\section{CONCLUSION}

La promotion de l'inclusion financière est devenue un objectif majeur pour Bank Al Maghrib. Une stratégie pour l'inclusion financière a été adoptée pour le développement du secteur financier qui comprend plusieurs mesures liées à la promotion des services bancaires pour les populations à faible revenu. Plusieurs études ont montré qu'un secteur financier inclusif est essentiel pour réduire la vulnérabilité des ménages à faible revenus et pour encourager l'essor des petites et moyennes entreprises à travers une meilleure répartition de capitaux. Notre étude a démontré que l'utilisation du mobile money est un levier d'inclusion financière et d'accès aux services financiers durant la crise sanitaire du Covid-19. En effet, cette étude a montré qu'avant la crise sanitaire les personnes de sexe masculin sont les principaux utilisateurs du mobile money pour réaliser leur transactions chose qui est confirmée par les tests de la force de relation qui a indiqué que la variable Genre a une influence sur l'utilisation de mobile money avec une influence moyenne. Par contre durant la pandémie du Covid-19, les variables genre et revenu, influencent significativement sur l'utilisation de mobile money. Il faut donc sensibiliser le public à l'importance des services financiers, Intégrer davantage les femmes dans le système financier à travers l'utilisation de paiement mobile, la 
généralisation de l'éducation financière, car elle contribue à l'amélioration de leur niveau de vie en leur permettant de mieux comprendre ces services et à mieux gérer leurs revenus et leurs économies.

Cette étude nous a permis de nous rendre compte que même si les réformes financières entreprises n'ont pas abouti entièrement aux résultats escomptés, elles ont été propices au développement de l'inclusion financière au Maroc.

\section{Bibliographie}

[1] Aguera P. «inclusion financière, croissance et réduction de pauvreté », p : 19.

[2] Amegnaglo C J, «Analyse exploratoire de l'effet de l'émergence des services de compte de monnaie électronique (Mobile Money) sur l'inclusion financière au sud du Bénin », $\mathrm{p}: 21$.

[3] Arestoff.F et Baptiste $\mathrm{V}$, « Impact du mobile Banking sur les comportements d'épargne et de transferts à Madagascar », $\mathrm{P}: 52$.

[4] Ashenafi F, «The Role of Mobile Money in Financial Inclusion in the SADC Region», 2016. https://doi.org/10.13140/RG.2.2.26994.71369.

[5] Aucante $\mathrm{M}$, «La bancarisation de l'Afrique par les téléphones mobiles: de nouveaux acteurs sur la scène financière mondiale? », p : 164.

[6] Banque Européenne d'investissement «Le secteur bancaire en Afrique subsaharienne - Évolutions récentes et inclusion financière numérique $», p: 190$.

[7] Bélisle $\mathrm{M}$ «Accessibilité financière des individus et rôle de la microfinance au Ghana et en Tanzanie », p : 144.

[8] Berthaud A et Gisela D. «Panorama de l'inclusion financière postale à travers le monde Modèles d'activité et questionsclés », p : 99.

[9] Bidiasse H et Gregory P M, «Les déterminants de l'adoption du mobile money : l'importance des facteurs spécifiques au Cameroun ». Revue d'économie industrielle, no 165 (15 mars 2019): 85-115. https://doi.org/10.4000/rei.7845.

[10] Bouhriz Daïdj A Ikhlef.A, «Innovations technologiques des services bancaires », P : 173.

[11] Boukhatem J et Mokrani B, «Effets directs du développement financier sur la pauvreté : validation empirique sur un panel de pays à bas et moyen revenu». Mondes en développement $\mathrm{n}^{\circ} 160$, no 4 (2012): 133 . https://doi.org/10.3917/med.160.0133.

[12] Bounie D et Marc B, Abel F, et Marianne V, «La détention et l'usage des instruments de paiement en France ». Revue d'économie financière 91, no 1 (2008).p: 53-76. https://doi.org/10.3406/ecofi.2008.5057.

[13] Calderon C, Gerard K, Vijdan K, Megumi K, et Catalina C C « Une analyse des enjeux façonnant l'avenir économique de l'Afrique ». The World Bank, 2019. https://doi.org/10.1596/978-1-4648-1510-2.

[14] Chaix L, «Le paiement mobile : modèles économiques et régulation financière ». Revue d'économie financière 112, no 4 (2013): 277. https://doi.org/10.3917/ecofi.112.0277.

[15] Chaix L, et Torre $\mathrm{D}$, « Le double rôle du paiement mobile dans les pays en développement ». Revue économique 66, no 4 (2015): 703. https://doi.org/10.3917/reco.664.0703.

[16] Chencheh $\mathrm{O}$, «Les déterminants de l'adoption du e-banking par les institutions financières et la clientèle organisationnelle, et son impact sur l'approche relationnelle : cas de l'internet-banking en Tunisie », p :175.

[17] David A. Réduire les inégalités. Agence française de développement, 2019. https://doi.org/10.3917/afd.anda.2019.01.

[18] Demirguc-Kunt A, Klapper L, Dorothe S, Ansar S, et Hess J. The Global Findex Database 2017: Measuring Financial Inclusion and the Fintech Revolution. Washington, DC: World Bank, 2018. https://doi.org/10.1596/978-1-4648-1259-0.

[19] Dissaux T , «Socioéconomie de la monnaie mobile et des monnaies locales au Kenya : quelles innovations monétaires pour quel développement? » Revue de la régulation, no 25 (3 juillet 2019). https://doi.org/10.4000/regulation.15139.

[20] Fox M, et Droogenbroeck V N, «Les nouveaux modèles de mobile Banking en Afrique: un défi pour le système bancaire traditionnel ? » Gestion 2000 34, no 5 (2017): 337. https://doi.org/10.3917/g2000.345.0337. 
[21] Gruber H, et Pantelis K, « Mobile Telecommunications and the Impact on Economic Development: MOBILES AND GROWTH ». Economic Policy 26, no 67 (juillet 2011): 387-426. https://doi.org/10.1111/j.1468-0327.2011.00266.x.

[22] Haoudi A, et Rabhi R, «Les déterminants de de l'inclusion financière en Afrique: Évidence sur la détention d'un compte courant », P : 13 .

[23] Hoby R, « Mémoire pour l'obtention du Diplôme de Licence ès-Sciences Economiques », p :33.

[24] Hourriez J M, et Roux V, «Vue d'ensemble des inégalités économiques », p : 60.

[25] Ilahiane H, « ECONOMIC AND SOCIAL EFFECTS OF MOBILE PHONE USE IN MOROCCO », 2021 , p : 15.

[26] Jack W, et Suri T, «Risk Sharing and Transactions Costs: Evidence from Kenya's Mobile Money Revolution». American Economic Review 104, no 1 (1 janvier 2014): 183-223. https://doi.org/10.1257/aer.104.1.183.

[27] Kiendrebeogo Y, et Minea A. «Accès aux services financiers et réduction de la pauvreté dans les PED ». Revue économique 64, no 3 (2013). https://doi.org/10.3917/reco.643.0483.

[28] Kuindja N, «Impact d'une inclusion financière pérenne des populations sur la rentabilité des EMF : Proposition d'un modèle conceptuel », no 4 (2020).p :22.

[29] Ladli A. « Les moyens de paiement électronique: «La carte CIB au niveau de CNEP Banque agence de Tizi-Ouzou » », $\mathrm{P}: 94$.

[30] Mercanti-Guérin M. «Crise du secteur bancaire et portrait de la banque idéale: une étude menée auprès des jeunes consommateurs ». La Revue des Sciences de Gestion 249-250, no 3 (2011),p :57. https://doi.org/10.3917/rsg.249.0057.

[31] Ndiaye A, et Weibigue A. «Mobile-Banking, un potentiel d'inclusion financière au Sénégal : le rôle de la proximité géographique des points de services de transfert d'argent sur l'adoption », p : 18.

[32] Ngono J F L. «L'indice d'inclusion financière à l'heure du mobile money » 11 (2020): p :12.

[33] Piffaretti N. «monnaie electronique, monnaie et intermediation bancaire », p : 239.

[34] Pinos F. «Inclusion financière et populations précarisées: effets des business models des services financiers en France $», \mathrm{p}: 401$.

[35] Rapport 2020 « la pauvreté et la prospérité partagée revers de fortune », 2020,p: 24.

[36] Tabi N. « Mobile money et inégalités de revenus en Afrique Subsaharienne » 2, no 4 (2020), p :22.

[37] Tangakou Soh R. «L'inclusion Financiere Et Le Paiement Mobile En Zone CEMAC ». European Scientific Journal ESJ 15, no 7 (31 mars 2019). https://doi.org/10.19044/esj.2019.v15n7p101.

[38] World Bank. «East Asia \& Pacific ». In Global Economic Prospects, June 2015: The Global Economy in Transition, par World Bank, p: 107-17. Global Economic Prospects. The World Bank, 2015. https://doi.org/10.1596/978-1-46480483-0_ch2_EAP. 